\title{
Communicating Reproductive Coercion in the Context of Domestic and Family Violence: Perspectives of Service Providers Supporting Migrant and Refugee Women
}

\author{
Nicola Sheeran ${ }^{1}\left[\right.$ [ Laura Tarzia ${ }^{2,3} \cdot$ Heather Douglas $^{2}$
}

Accepted: 8 January 2022 / Published online: 12 January 2022

(c) Crown 2022

\begin{abstract}
The current study explored the language barriers to help-seeking in the context of reproductive coercion and abuse (RCA), domestic and family violence (DFV), and sexual violence (SV), drawing on observations by key informants supporting women from migrant and refugee communities. A lack of shared language has been identified as a key barrier to help seeking for migrant and refugee women experiencing DFV more broadly, though how language intersects with help seeking in the context of RCA is yet to be investigated. We conducted 6 focus groups with 38 lawyers, counsellors, and social workers supporting women experiencing DFV in Brisbane and Melbourne, Australia. Our findings address two main areas. First, consistent with past research in DFV, our participants identified language as a barrier for women when communicating about sexual and reproductive issues in the context of health and police encounters. More specifically, our findings suggest that the inability of health professionals and police to communicate with women who have low or no English proficiency not only negatively impacted victims/survivors' ability to access support, but also facilitated the perpetration of RCA. We conclude that language can be a mechanism through which coercive control is enacted by perpetrators of RCA and health and policing systems may not be equipped to recognise and address this issue. We also suggest that greater conceptual clarity of RCA is needed within the DFV sector in order to tailor responses.
\end{abstract}

Keywords Reproductive coercion $\cdot$ Violence against women $\cdot$ Qualitative $\cdot$ Migrant women $\cdot$ Australia $\cdot$ Communication

\section{Introduction}

Reproductive coercion describes a range of behaviours that deliberately compromise a woman's reproductive autonomy, including coercing a woman to become pregnant, to terminate or continue a pregnancy, to take contraception unknowingly or against their will, and/or contraception sabotage (Clark et al., 2014; Miller et al., 2010). Reproductive coercion is typically perpetrated by intimate male partners (Grace \& Anderson, 2018), though family members

Nicola Sheeran

n.sheeran@griffith.edu.au

1 School of Applied Psychology, Griffith University, Mt Gravatt Campus, Messines Ridge Road, Mt Gravatt, QLD 4121, Australia

2 University of Melbourne, Melbourne, Australia

3 Royal Women's Hospital (Centre for Family Violence Prevention), Parkville, Australia may also be perpetrators (Silverman \& Raj, 2014). Robust prevalence data is lacking but international research suggests rates of 5-30\% (Grace \& Anderson, 2018; Rowlands $\&$ Walker, 2019). In recognition of the context of fear and control that typically accompanies reproductive coercion, as well as the overt physical and sexual tactics (in addition to coercive behaviours) that are often used, Tarzia and Hegarty (2021) argue that reproductive coercion and abuse (RCA) is a more appropriate term.

RCA is argued to be a distinct form of abuse that intersects with other forms of domestic and family violence (DFV) and sexual violence (SV) (Tarzia \& Hegarty, 2021) and is often part of a broader pattern of coercive control (Stark, 2007; Stark \& Hester, 2019; Tarzia \& Hegarty, 2021). However, there is a lack of conceptual clarity and significant overlap between the behaviors that consitute RCA and those defined as intimate partner/family violence or sexual violence. Intent may be central to disentagling RCA from these other related forms of violence (Tarzia \& Hegarty, 2021), yet intent may not be clear: either to the victim-survivor or 
to those supporting her. For example, removing a condom during sex could be classified as intimate partner/family violence, sexual assault or reproductive assault depending on the nature of the relationship and whether the removal was for pleasure or to cause a pregnancy. Similarly, punching or kicking that accidently versus deliberately causes a miscarriage could classified as intimate partner/family violence, RCA, or both. Perpetrators of RCA harness sexual violence to promote pregnancy, and physical and psychological violence to either promote or prevent pregnancy as well as coercive and controlling behaviours to reduce reproductive autonomy (Tarzia \& Hegarty, 2021). Given the complexities of defining RCA and debates about the centrality of intent, we explore RCA broadly in the context of SV and DFV.

International research suggests that RCA may be experienced more frequently by women who are Black, Latina, or multiracial, compared to white (Clark et al., 2014; Grace \& Anderson, 2018; Holliday et al., 2017), though these studies should be interpreted with caution due to definitional and measurement issues. In Australia, Price et al (2019) found that women reporting RCA were more likely to identify as Aboriginal or Torres Strait Islander or culturally and linguistically diverse (CALD) (Price et al, 2019). Similarly, research on DFV more broadly has suggested a range of individual, community and societal factors intersect to increase the risk of violence for women from some cultural backgrounds (Ames Australia, 2016).

Australia is one of the most multicultural and multilingual nations in the world with nearly half (49\%) of the population born overseas or with one or both parents born overseas; many from non-English speaking countries (e.g., China, Philippines, Vietnam, Italy, Malaysia, Germany, North Africa, Middle East, las Americas; Australian Bureau of Statistics [ABS], 2021). While Australia's official language is English, over 300 languages are spoken nationally. Only 40-50\% of migrants report speaking English very well, with 2-3\% not speaking English at all (ABS, 2021), suggesting many migrants and refugees from non-English speaking backgrounds have low-English proficiency. Having lowEnglish proficiency creates additional complexities for those who experience DFV (Alliment \& Ostapiej-Piatowski, 2011; Vaughan et al., 2016), as they often lack a shared language with service providers.

Language diversity poses a significant challenge to the provision of all kinds of human services (Minas et al., 2013) and can obstruct people's access to information about available services or to navigate systems, and interpreters and written information in other languages are often scarce (Bonar \& Roberts, 2006). Communication issues are often the most immediate barriers to a woman leaving an abusive relationship (Lemon, 2006; Vaughan et al., 2016) and language is a known systemic barrier impacting help-seeking and reporting more generally in the context of DFV and
SV (Alliment \& Ostapiej-Piatowski, 2011, Mitra-Kahn et al., 2016; InTouch, 2010; Tam et al., 2016, Taylor \& Putt, 2007). Given the intersections between DFV, SV, and $\mathrm{RCA}$, it is likely that language is a barrier to help-seeking and reporting for migrant and refugee women experiencing RCA, though no research has directly explored this. More so, while there have been advances in how DFV and SV are raised and discussed, there does not yet appear to be an accepted language among support workers to discuss RCA (Douglas et al., 2021; Tarzia et al., 2021; Wellington et al., 2021). As such, a range of factors may influence migrant and refugee women's ability to identify RCA and seek legal and social support and they may have markedly different experiences compared to non-migrant and refugee women because of language diversity. Thus, there is a need to understand how language diversity intersects with a women's ability to seek help when they are experiencing RCA in the context of DFV/SV (Alliment \& Ostapiej-Piatowski, 2011). This paper addresses that gap by exploring service provider perspectives of the experiences of migrant women from linguistically diverse backgrounds who experience RCA in the context of DFV/SV in Australia. Our guiding research question was "what role does language play in help-seeking for migrant and refugee women experiencing domestic and family violence that may include reproductive coercion and abuse/sexual violence". We draw on data generated in focus groups with key informants (service providers working in DFV services) to explore the intersection between language, RCA, and help-seeking for migrant and refugee women. Key informants were chosen as they can identify issues pertaining to individual clients, as well as issues across clients, while also being able to share their own perspectives on how they communicate about RCA (Dingwall, 1997).

\section{Method}

Participants and Procedure Thirty-eight staff from six DFV services participated in one of six focus groups (see Table 1 for a breakdown of participant distribution across services). Eleven were lawyers, one was a paralegal worker and 26 were social workers or counsellors. All participants in the study were female, reflecting the demographic profile of workers in this area. Although we did not collect information about participants' cultural identity/linguistic background, the two services for immigrant/refugee women were primarily staffed by workers who were themselves from diverse cultural and/or linguistic backgrounds. We acknowledge that culture is not fixed or innate to any individual or group of individuals (AMES Australia and Australian Government Department of Social Services, 2016) and we use the term "culture" or CALD only when our participants do. Our participants appeared to define culture as region of origin (Raj 
Table 1 Focus group details

\begin{tabular}{lllll}
\hline FG \# & State & Type of Service & $\begin{array}{l}\text { Number of } \\
\text { participants }\end{array}$ & Type of practitioners \\
\hline 1 & QLD & Domestic violence (general) & 7 & Counsellors/social workers \\
2 & QLD & Legal & 5 & Lawyers/social workers \\
3 & VIC & Legal & 9 & Lawyers/social workers \\
4 & QLD & Domestic violence (immigrant-non- & 6 & Counsellors/social workers \\
& & English-speaking backgrounds) & & Counsellors/social workers \\
5 & VIC & Domestic violence (refugee/migrant) & 5 & Counsellors/social workers \\
6 & VIC & Domestic violence (general) & 6 & \\
\hline
\end{tabular}

$F G$ Focus group, $Q L D$ Queensland, VIC Victoria

\& Silverman, 2002). All the participants worked in non-governmental agencies that delivered services to women who have experienced DFV, including significant proportions of women from non-English speaking backgrounds. Three of the focus groups took place in Melbourne and three in Brisbane. The sites were selected for this initial study because the legal definitions of DFV are similar in both states. ${ }^{1}$ Services included a specialist DFV legal service, a specialist DFV service for migrant/refugee women and a general DFV support service who provided support to those from migrant and refugee backgrounds in each city.

Service directors were sent invitations by the researchers to participate in the focus groups via email along with an information sheet about the project. Potential service participants were asked to contact the researchers if they wanted their service to take part in the focus groups. Directors of services then invited participants to the focus group. Eligibility criteria included being employed by the service to assist women to prepare domestic violence orders and/or provide women with information about legal options. On the day, participants provided written informed consent. Each focus group ran for approximately $60-90 \mathrm{~min}$. The focus groups were audio-recorded and transcribed. Participants were asked what they thought RCA was (including behaviours that their clients had reported), how they broached it with clients, and how they worked with disclosures. In answering these questions participants spontaneously raised issues related to language and barriers to help-seeking. Additional questions focussed on current legislation and whether it was adequate (see Douglas et al., 2021 for further findings). Participants drew on their observations of interactions they had witnessed as well as accounts provided by the women they were supporting. These participants were chosen as key

\footnotetext{
${ }^{1}$ In Queensland and Victoria civil protection order legislation defines domestic violence widely to include coercive and controlling behaviour. See Sect. 8, Domestic and Family Violence Protection Act 2012 (Qld); Sect. 5 Family Violence Protection Act 2008 (Vic).
}

informants because they are first responders and have an important role to play in advocating and supporting women who experience RCA.

Analytic Strategy The transcripts were manually coded by the authors and analysed using reflexive thematic analysis from a social constructivist paradigm and drawing on phenomenology to focus on the way our participants co-created meaning by sharing their experiences supporting survivors/ victims of RCA (Braun \& Clarke, 2006, 2019). Initial coding steps included familiarisation with the data, followed by development of descriptive codes across focus groups, before themes were generated through a process of interpretive coding where descriptive codes were brought together and underlying patterns or meanings were teased out before being situated into the extant literature (Braun \& Clarke, 2006, 2019).

Ethical Considerations Ethics approval for the study was granted by Griffith University human ethics committee (GU ref number: 2019/578). To ensure confidentiality of participants' names and organisations, quotes are identified only by the number given to the focus group (i.e., FG1).

\section{Findings}

The aim of the current research was to explore the way that communication impacts on help-seeking in the context of RCA, SV, and/or DFV for migrant and refugee women. Our findings are based on accounts of lawyers, counsellors and social workers who support, advocate, and represent women who have experienced RCA and/or DFV and have engaged with health, police, or court systems and cannot be understood as a literal description of these systems as a whole. Our findings suggest that language created particular barriers to addressing RCA in two main contexts: health and police encounters. Below we present two themes that explore how language was exploited in ways that allowed perpetrators to exert control and enact RCA in these contexts: 
1) "Communication is good enough for us"; and 2) "I will speak on her behalf". We discuss each of these themes in turn highlighting the core issues our participants identified and the implications they identified for women from nonEnglish speaking backgrounds who had low-English proficiency. First, we overview two subthemes that contextualize our findings.

\section{Contextualizing Findings}

The first contextual finding raised by our participants was their belief that while most women would find it difficult to discuss RCA, expectations around privacy contributed to some migrant women being reluctant to discuss sexual and reproductive matters, particularly with members of the another gender, "it's difficult just in general to make those disclosures, but then when you've got other cultural considerations there, that's an added maybe reason why people don't speak of it as well." (FG2). The appropriateness of discussing RCA with a man was raised as a barrier in police, courtroom, and health encounters.

That's been my experience with certain communities, being so intimate and them not wanting to talk about [sexual and reproductive abuse]...often if they've gone to report it to police and it's a male officer, or there are men in the courtrooms, it's not for a male doctor, it's completely inappropriate to talk about any sexual reproductive health issue with a male. (FG3)

Thus, the gender of the communication partners and the content of the disclosures could impact on women's ability to disclose RCA.

The second contextual finding was that our participants identified that navigating the system to report any form of DFV and pursue legal applications was a highly complicated process, particularly for migrant and refugee women. In particular, they noted that women from diverse backgrounds were left vulnerable to a system they did not understand, "then when you add in our CALD clients, there's just that added layer of complication that comes with that as well... vulnerability, and when you're vulnerable, even just being able to call the police becomes difficult" (FG2). Previous research has highlighted how people from some migrant and refugee backgrounds find it difficult to trust people in positions of power (such as police), often because of experiences in their country of origin (In Touch, 2010). Our findings similarly suggest that seeking help may place some women from migrant and refugee backgrounds in a position of vulnerability in a system that they may not understand, thus deterring help seeking. With these contextual findings in mind, we discuss our main themes as relevant to our research question.

\section{Communication is Good Enough for us}

One of the key findings reported by participants in all agencies was their belief that health professionals and police fail to use interpreters because communication was 'good enough' for them. However, participants reported that, for the women experiencing RCA, the failure to use interpreters had implications for the content of statements and disclosures and therefore for the accuracy of statements and disclosures. That communication may not be good enough for the woman did not appear to be considered.

When discussing their clients' contacts with health professionals, participants primarily identified general practice as the most problematic context. They reported that physicians in general, and GPs specifically, were unlikely to use an interpreter, "So, going to a GP...some of them have to take family members because GPs have not the time to call an interpreter, as the duration is only for $20 \mathrm{~min}$ each person" (FG4). As highlighted in this quote, women may feel pressured to take a family member to communicate with their physician. Participants also noted an unwillingness on the part of the provider to engage interpreters, even when interpreter services were available: “[GPs] said they don't have this [interpreter] service available and even if I give them the contact number to ring an interpreter at that appointment, they refuse to do it"(FG5). Participants reported that systems needed to be in place to support access to interpreters, such as embedding interpreters in services or making interpreters compulsory. Others noted that even in hospitals where there are policies around interpreter use, particularly when gaining informed consent, they often were not used.

the doctors can be very poor at using the phone interpreter services. ... They're like, we don't have time.

Even though the hospital actually has policies. They

have - you can get an interpreter in that hospital very easily. (FG1)

While a range of motivations for not using interpreters was raised, including lack of time, lack of knowledge, lack of resources (like access to a telephone), and not feeling it is something they 'do', what also emerged was a sense that communication was 'good enough' from the physician or police officer's perspective. "I prepped a client to ask the police to get an interpreter. She asked and then they declined and said, "you can speak English well enough for us to understand you"." (FG1). As highlighted in this quote, our participant believed that the police were primarily focussed on whether they could understand the woman and the use of an interpreter was their choice, rather than the woman's. This raises the question of whether the police had considered that she may need support to understand them, or whether she had sufficient vocabulary in English to communicate 
the complex behaviours and emotions that underpinned her experiences of DFV or RCA.

While most participants noted that they felt the lack of access to interpreters was problematic, some also noted that the use of interpreters was not without its own issues. For example, participants in one focus group noted: "[the] lack of availability of female interpreters and particularly in small groups of-small communities where there are maybe one or two interpreters for the entire language group" (FG1) was an issue, highlighting that access issues are not purely due to the service provider being unwilling to engage interpreters. Another participant further highlighted that the interpreter could also be a known member of the community, which could also create barriers to use, compromising the effectiveness of the communication for the person seeking help.

Communicating about a complex issue like RCA requires nuanced language to communicate coercive and controlling behaviours and how the victim feels. As highlighted below, the nature of behaviours that may constitute RCA may not be easily communicated.

Participant 1: .... [Police] don't engage interpreters and there was one occasion ...we attended an interview and [the case worker] had to explain some of the terminology that the police was using.

Participant 2:Ejaculation.

Participant 1:Yeah. For instance... they had to be explicit [non-verbal/hand gestures] about it so the woman could understand.

Participant 2:So, if our caseworkers were not there, [the] woman just wouldn't know.

Participant 1:She would have said yes or no to something that... She might have said, no, that didn't happen. (FG4)

Thus, specialised training may be required to ensure that our workforces have both linguistic and cultural competence so that they can facilitate effective communication and advocate for appropriate interpreters for victims-survivors where English is not their first language.

\section{I will Speak on her Behalf}

Our participants felt that the lack of use of qualified interpreters in health and police encounters created a void whereby perpetrators could position themselves as the interpreter for the woman, which we argue, could facilitate RCA and other forms of violence. By enabling the perpetrator to act as the interpreter, rather than a qualified independent interpreter, there is the potential for health professionals to facilitate abuse as they allow the perpetrator to be the only voice, ultimately giving perpetrators control over reproductive decisions. Our participants highlighted that the use of the husband as an interpreter was rarely questioned in health contexts, particularly by the GP. The main motivation for positioning themselves as the interpreter was perceived to be so that the perpetrator could control the information provided to the GP.

Participant 1: Yeah, the partner positioning themselves as being the go-between, and then relaying what information they want.

Participant 2: Usually, that's their way of controlling what information goes out, because obviously a doctor is a mandatory reporter as far as abuse towards children, but also, if a woman is showing up with injuries, and needs to speak to a doctor, or is hospitalised, he obviously wants to act as the interpreter. (FG3)

This strategy was reportedly used whether the migrant women could speak English or not, "one of my clients, she speaks perfectly fine English, she doesn't need an interpreter but [her] husband went into the sessions and told the GP directly, my wife doesn't speak English at all, so you tell me" (FG4).

The above example would be considered a significant medical failing as the physician did not independently assess the patient's language proficiency and instead trusted the perpetrator (who was not his patient).

Participants noted that the lack of access to interpreters and use of perpetrators as interpreters by health professionals lead to outcomes that constitute RCA. Specifically, one participant noted that a woman had a hysterectomy performed without her knowledge after her husband instructed the physician, ultimately ending her reproductive choices, "Yeah. There was a ...medical procedure performed on one of our clients without her consent or knowledge until after the fact. (FG 2)". Another participant discussed how the perpetrator had arranged a prescription for contraception with the physician without her knowledge. "The women have been made to take something, like vitamins, but it was really a contraception (FG 4)". Here the perpetration of RCA was directly facilitated by the perpetrator being able to control information. As highlighted by participants in FG3, "Yeah, just because he's doing all the interpreting. He can say whatever he likes, basically... 'She doesn't want-you know, she doesn't want all of these sorts of things [contraceptives]. Doesn't matter what she says" (FG4). Thus, it appears that language barriers (and non-use of qualified and appropriately trained interpreters) may not only be a tool for limiting the woman's capacity to disclose RCA but may also give perpetrators control over reproductive choices.

While most participants spoke of issues in health encounters with physicians, one group of participants also raised language issues that had occurred with allied health professions such as counsellors and psychologists. 
Or to actually be referred to counselling in the context of her suffering from postnatal depression. The perpetrator attends the whole time to make sure she does not disclose the context of family violence. Then his excuse is that she doesn't understand, so I'm going to be here, you don't need to provide an interpreter. I will talk on her behalf because I am the partner. (FG3)

We ...tried to ...get the ...psychologist report to support her visa application and ...immigration [were] actually questioning the family violence... There is a record that she actually had been attending counselling the whole time throughout the relationship ... But why was family violence never actually mentioned that this occurs? Because, of course, he actually attended every single appointment that she attended. (FG3)

These examples show how a perpetrator can silence victims-survivors by both talking on her behalf and by his presence. Our participants also noted examples of where sons were sent along by perpetrators to their mothers' health appointments. Our participants then highlighted how difficult it would be for their clients to discuss reproductive issues as it would be inappropriate discussing this with a male relative present, as highlighted by participants in FG2:

Participant 1: I know of quite a few of the clients that I've had, they've had to go to a doctor with their partner. This is particularly true when they don't speak English... Where they either get taken by their current partner or by their older sons.

Facilitator:Okay, and [act] as interpreters too?

Participant 2: Yes.

Participant 1:Interpreters, yeah. Imagine talking through ... with your son there about reproductive - I don't think it's going to happen.

Participant 2:Doesn't happen.

Also of concern to our participants' were reports by their client's that police had used the perpetrator either as an interpreter, or as the person from whom they took the only statement about events involving the woman when conducting investigations. For example, "there's another case recently where the police used the perpetrator to be the interpreter... because he had better English than she did, so they were getting him to interpret her. So of course their statement was compromised" (FG1).

Compromised statements may mean that the incident is minimised resulting in a lack of response by police.

We have a lot of cases, that police go to their places and ...then, because of different language barriers .... So, then they only interviewed the perpetrator, and he told them that she hurt herself, and that nothing hap- pened, and they put that in their report, and they just went home. (FG4)

Participants noted that one of the risks to women when the perpetrator interpreted for them or made the only statement to police, was that the woman experiencing abuse could be misidentified as the perpetrator and may become the respondent in a protection order.

I was also referring to the number of clients that aren't offered interpreters when they're calling the police or giving their side of the story and how that just does not work in their favour. The amount of times CALD women call the police and the police speak to the English speaker who's the man and find themselves the respondents of orders, that's it. That's just because they can't speak English as well as him. (FG2)

Overall, this theme highlighted our participants impressions of how system failures and a lack of clear protocols that ensured that their clients with low-English proficiency had access to a professionally trained interpreter created a void that allowed perpetrators to control information.

\section{Discussion}

The current study explored the role that language plays in help-seeking for migrant and refugee women experiencing RCA and other forms of violence and found that there may be an under-utilisation of qualified interpreters by health professionals and police that allows perpetrators fill the void by acting as interpreters. Our findings suggest that once in the role of interpreter, perpetrators can then control the information that is sent and received. We argue, that in the context of reproductive coercion and other forms of violence, the act of using a perpetrator or delegated family member risks facilitating the perpetuation of the abuse both directly and indirectly. For example, controlling the information that is disclosed and received means that a woman could miss out on access to abortion or certain forms of contraception, reducing her reproductive autonomy. Past research has also shown that failure to engage interpreters can make women feel that their voices and experiences are diminished, that the law is biased towards the male perpetrator, which ultimately increases marginalisation (InTouch, 2010). Though increasing the use of qualified interpreters is part of the solution, the confusion that exists about what RCA is and when and how it intersects with sexual violence means that specific training for interpreters will likely be necessary to ensure they have had education about RCA and other forms of violence to facilitate discussions and disclosures.

Despite policies that state that qualified interpreters should normally be used, and free access to telephone 
interpreters in health consultations (Phillips, 2010), research suggests there is a significant underuse of qualified interpreters in police and health encounters (Atkin, 2008; Gray et al., 2011; Wakefield et al, 2015), with family members frequently used instead (Atkin, 2008; Pines et al, 2020) often because it is more convenient (Diamond et al, 2009). Gray et al. (2011) found that family members were used in $49 \%-83 \%$ of general practice consultations. Research suggests that using a family member as an interpreter in health contexts brings with it a range of risks including poorer clinical outcomes (Karliner et al., 2007) and lower patient satisfaction (Garcia et al., 2004). However, there are also proponents who suggest family members can be appropriate interpreters and exploring when this might be appropriate (Hilder et al, 2017). An important consideration is how a clinician would assess whether the patient was experiencing RCA or other forms of abuse and whether the support person was a perpetrator. In the absence of evidence-based risk assessment processes, and in line with research that suggests that formal interpreters should be used to help patients from a refugee background discuss trauma and build trust with their doctor (Gray et al, 2011), we suggest formal interpreters are essential to allow women to have voice and control over their own reproductive choices. Further the complexity of RCA makes the underuse and inappropriate use of interpreters especially problematic in health contexts, where health professionals may be the first point of contact for a woman experiencing RCA, impacting both their ability to disclose the abuse and the quality of the disclosure, and ultimately impacting the kind of advice, treatment, and referral women receive.

Even in hospitals where there has been a targeted strategy to make access to interpreter services more effective, nearly half of all patients with low-English proficiency visiting hospitals in Queensland in the last 12 months did not receive a qualified interpreter (Gold Coast Hospital and Health Service [GCHHS], 2017). Research suggests a similar situation in general practice (Atkin, 2008; Huang \& Phillips, 2009). When asked, most health professionals note a range of practical barriers (i.e., challenges accessing services outside of business hours, perceived or actual costs, time consuming etc.). Health practitioners also find it difficult to assess whether an interpreter is needed (Jones et al., 2019), though participants in the current study perceived that the health practitioner's attitude to engaging an interpreter may be an additional barrier (i.e., "they just don't want to do it"). We suggest further research is needed to unpack attitudes held by the professionals themselves.

A number of studies in police and health contexts have identified and tried to understand why interpreters are underused (Diamond et al., 2009; Dixon \& Travis, 2007; Gibbons, 1995). Research exploring the use of interpreters by police generally has found that police are often reluctant to use interpreters unless there are major communication problems and in cases where there are relatively minor offences (ALRC, 1992; Gibbons, 1995). Police often underestimate the complexity of words and phrases in police interviews and have difficulty recognising the level of English someone requires to participate in police interviews (ALRC, 1992; Dixon \& Travis, 2007). Thus, they may not recognise that someone requires an interpreter. Our findings also suggested that some police may focus on ensuring they understand the woman 'enough' and may not be concerned about whether she could communicate the complexity of her experience or understand the police. Thus, it seems that some police may also have a narrow view of communication and the role of interpreters. This view has been found in doctors, where the use of interpreters allowed the doctors to tell the patient what would happen, rather than allowing the patient to elicit information from the doctor (Jones et al, 2019). We argue that the two-way flow of information is vital to ensuring accuracy in reports and facilitating the understanding of the woman experiencing RCA. There also appears to be a general lack of linguistic competence and understanding of how communicating in a non-native language can affect the nature of statements made.

Work in the field of psychology has shown that conducting an initial assessment in a person's non-native language affects the recounting and retrieval of traumatic, autobiographical, and general memories (Javier, 1995; Schwanberg, 2010). This is likely also true in police and medical contexts, which could impact on the content of the statement made by the woman. Similarly, people communicating in their non-native language often have a smaller vocabulary and lower lexical access (particularly for abstract words), even when fluent (Bialystok \& Craik., 2010). Communicating in a non-native language increases anxiety and affects prosody, rate, latency, and intonation of speech (which help to convey emotions) (Bialystok \& Craik., 2010; Dewaele \& Costa, 2013; Garcia de Blakeley et al., 2017), which can result in fewer displays of emotion, increased detachment and intellectualising of content, and shorter narratives (Pavlenko, 2006). As such, an assessment of "fluent enough" may be incorrect and in such cases, the potential psychological, cognitive, and social effects of language diversity may be overlooked, impacting the accuracy and quality of the woman's statement and the impression that is formed (Smith \& Skinner, 2017).

Recent research has highlighted the importance of linguistic competence (and not just cultural competence) for mental health workers (Garcia de Blakeley, 2020). Our findings suggest that police and health professionals more generally may benefit from improved linguistic competence, which includes awareness, knowledge, and 
skills in managing language diversity. Given that behaviours that constitute RCA (and coercive control in general) can be difficult to articulate and identify, limited vocabulary and capacity to express a range of emotions could significantly impact whether claims are able to be communicated, understood, and responded to. Rather than situating the issue within the woman and arguing that it is her responsibility to be able to communicate with the doctor, we and others argue it is the doctor's responsibility to ensure they can communicate with their patients. A recent paper suggested that doctors should be trained during medical school in a second language to allow them to provide language-concordant care to their patients (Molina \& Kasper, 2019). At the very least, health professionals should have linguistic competence. Our research suggests health professionals may be failing their patients by not using properly trained interpreters.

\section{Implications for Policy, Practice and Future Research}

Our study joins a plethora of previous studies highlighting the importance of engaging formal interpreters to support migrant and refugee women experiencing DFV and/or SV who have low-English proficiency (Alliment \& OstapiejPiatowski, 2011; InTouch, 2010; Lemon, 2006; MitraKahn, et al., 2016; Tam et al., 2016; Taylor \& Putt, 2007; Vaughan et al., 2016). Concerningly, despite decades of advocacy and research highlighting this issue, our findings suggest interpreters are still underutilised. While the study was conducted in Australia, the findings have relevance to many other countries where systems are similarly problematic. Thus, more work is needed to embed accessible interpreter services into health and police services and ensure their use. Our findings suggest that health professionals and police should be trained in RCA and other forms of abuse and the importance of using qualified interpreters. In particular, we need to raise awareness of the need to have female police or health providers who communicate with migrant and refugee women on issues of RCA. The appropriateness of discussing sexual and reproductive health issues with people of different genders has been raised repeatedly as a barrier to help seeking for women from different communities, across a range of contexts (i.e., legal proceedings, Judicial Council on Cultural Diversity, 2016). Services should ensure they have policies and procedures in place that ensure appropriate interpreters are routinely used.

Our findings also highlight the importance of training on RCA and other forms of abuse for interpreters but also social workers, psychologists and health professionals who serve migrant and refugee populations. Concerningly, our participants reported that some counsellors and psychologists had failed to use interpreters and that they may not have assessed their client's safety. Although there are guidelines around interpreter use for these professions (i.e., Australian Psychological Society, 2007) there is little research investigating the use of interpreters by psychologists and counsellors. Our findings suggest this should be investigated in future research.

While considerable progress has been made in the way we discuss sexual violence and the multiple ways in which DFV can be perpetrated, it does not appear that this language extends to RCA. We propose that there may not just be a literal language barrier for migrant and refugee women but also a metaphorical barrier for DFV sector workers because they do not have the language to differentiate RCA from SV and DFV. Thus, further training of people working in the DFV sector is needed to assist them to ask questions that might elicit any coercive or controlling behaviours perpetrators are using to control their reproductive choices. This would then allow appropriate referrals to be made (i.e., referral for a long-acting reversible contraceptive) but would also enable women to properly describe their experience.

Consistent with our finding that when a woman has low-English proficiency, the woman's statement may not even be taken by police, Ulbrick and Jago (2018) found that police failing to interview both parties and/or interview parties separately was a factor in misidentification of women as perpetrators. Nancarrow et al. (2020) highlight that although laws in some states of Australia require that police and courts identify "the person most in need of protection", when there are conflicting claims about who the perpetrator is, cross orders are commonly made. Perpetrators can also use legal processes as a tactic for further control and abuse, and women from migrant and refugee backgrounds may be at higher risk (Douglas \& Chapple, 2019). Thus, it is likely that women with low-English proficiency, who do not have access to interpreters, are at greater risk of being identified as perpetrators, having cross orders made against them, or not having protective orders put in place at all. While research has found that Aboriginal and Torres Strait Islander women are more likely to be subject to cross orders and labelled as perpetrators (Douglas \& Fitzgerald, 2018), there has not yet been systematic investigation of migrant and refugee women; a direction we suggest for future research. What is known is that misidentification results in a significant number of negative impacts that can result in reduced support and help seeking (BurgessProctor, 2012).

Finally, RCA is a relatively newly articulated form of violence and there is ongoing definitional debate. One issue that has received scant attention in the literature is where the threshold is for what constitutes RCA and whether there are differences in the experience of RCA in the context of DFV. 
More research is needed to articulate these boundaries and intersections.

\section{Limitations}

Our study utilised key informants who regularly work with migrant and refugee women who experience DFV. While they were able to share their experiences of interactions they had both witnessed and been told by victimssurvivors, we cannot be certain that victims-survivors felt the way participants described. Nor can we be certain that police officers or health professionals acted in the way that was reported or for the reasons that were attributed. Despite this limitation, language barriers were consistently raised by participants as a critical issue for the women they were supporting; an issue that can have serious consequences for both their sexual and reproductive health outcomes and court outcomes.

\section{Conclusion}

Underutilisation of interpreters is an ongoing issue for some survivors-victims of RCA and other forms of abuse from migrant and refugee communities. Medical and police contexts were identified as particularly problematic, though counsellors and psychologists may also fail to use interpreters. Professionals working in these contexts, as well as those working as interpreters and in DFV services may benefit from training on RCA and policies are required to ensure that perpetrators are not placed in a position where they can directly or indirectly control information and thereby reduce reproductive autonomy.

Funding Open Access funding enabled and organized by CAUL and its Member Institutions.

Open Access This article is licensed under a Creative Commons Attribution 4.0 International License, which permits use, sharing, adaptation, distribution and reproduction in any medium or format, as long as you give appropriate credit to the original author(s) and the source, provide a link to the Creative Commons licence, and indicate if changes were made. The images or other third party material in this article are included in the article's Creative Commons licence, unless indicated otherwise in a credit line to the material. If material is not included in the article's Creative Commons licence and your intended use is not permitted by statutory regulation or exceeds the permitted use, you will need to obtain permission directly from the copyright holder. To view a copy of this licence, visit http://creativecommons.org/licenses/by/4.0/.

\section{References}

Alliment, A., \& Ostapiej-Piatkowski, B. (2011). Supporting women from CALD backgrounds who are victim-survivors of sexual violence: Challenges and opportunities (ACSSA Wrap No.9). Australian Institute of Family Studies.

AMES Australia and Department of Social Services (Cth), Violence against women in CALD communities: Understandings and actions to prevent violence against women in CALD communities (AMES Australia and Department of Social Services (Cth), 2016).

Atkin, N. (2008). Getting the message across: Professional interpreters in general practice. Australian Family Physician, 37(3), 174-176.

Australian Bureau of Statistics. (2021). Migration, Australia. Canberra, Australian Capital Territory. https://www.abs.gov.au/ statistics/people/population/migration-australia/latest-release. Accessed 3/11/2021.

Australian Law Reform Commission. (1992). Multiculturalism and the law (Report No. 57).

Australian Psychological Society. (2007). APS Code of Ethics. Melbourne, Australia.

Bialystok, E., \& Craik, F. I. M. (2010). Cognitive and linguistic processing in the bilingual mind. Current Directions in Psychological Science, 19(1), 19-23. https://doi.org/10.1177/0963721409 358571

Bonar, M., \& Roberts, D. (2006). A review of literature relating to family and domestic violence in culturally and linguistically diverse communities in Australia. WA: Family and Domestic Violence Unit, Department for Community Development, Government of Western Australia.

Braun, V., \& Clarke, V. (2006). Using thematic analysis in psychology. Qualitative Research in Psychology, 3, 77-101. https://doi.org/10. 1191/1478088706qp063oa

Braun, V., \& Clarke, V. (2019). Reflecting on reflexive thematic analysis. Qualitative Research in Sport, Exercise and Health, 11, 589597. https://doi.org/10.1080/2159676X.2019.1628806

Burgess-Proctor, A. (2012). Backfire: Lessons learned when the criminal justice system fails help-seeking battered women. Journal of Crime and Justice, 35(1), 68-92. https://doi.org/10.1080/07356 48X.2011.631393

Clark, L. E., Allen, R. H., Goyal, V., Raker, C., \& Gottlieb, A. S. (2014). Reproductive coercion and co-occurring intimate partner violence in obstetrics and gynecology patients. American Journal of Obstetrics \& Gynecology, 210(1), 42.e1-8. https://doi.org/10. 1016/j.ajog.2013.09.019

Dewaele, J.-M., \& Costa, B. (2013). Multilingual clients experience of psychotherapy. Language and Psychoanalysis, 2(2), 31-50. https://doi.org/10.7565/landp.2013.005

Diamond, L., Schenker, Y., Curry, L., Bradley, E., \& Fernandez, A. (2009). Getting by: Underuse of interpreters by resident physicians. JGIM: Journal of General Internal Medicine, 24, 256-262. https://doi.org/10.1007/s11606-008-0875-7

Dingwall, R. (1997). Accounts, inerviews and observations. In Miller, G., \& Dingwall, R. (Eds), Context and Method in Qualitative Research (pp. 51-65). Sage Publications.

Dixon, D., \& Travis, G. (2007). Interrogating images. Audio-visually recorded police questioning of suspects. Sydney Institute of Criminology.

Douglas, H., \& Chapple, K. (2019). National domestic and family violence bench book. Retrieved July 7, 2020 from http://dfvbenchbo ok.aija.org.au/

Douglas, H., Sheeran, N., \& Tarzia, L. (2021). Reproductive coercion and legal recognition: Views of domestic violence workers. International Journal for Crime, Justice and Social Democracy, 9(3), https://doi.org/10.5204/ijcjsd.1704. 
Douglas, H., \& Fitzgerald, R. (2018). The domestic violence protection order system as entry to the criminal justice system for Aboriginal and Torres Strait Islander People. International Journal for Crime, Justice and Social Democracy, 7(3), 41-57. https://doi. org/10.5204/ijcjsd.v7i3.499

Garcia, E. A., Roy, L. C., Okada, P. J., Perkins, S. D., \& Wiebe, R. A. (2004). A comparison of the influence of hospital-trained, ad hoc and telephone interpreters on perceived satisfaction of limited English-proficient parents presenting to a pediatric emergency department. Pediatric Emergency Care, 20, 373-387. https://doi. org/10.1097/01.pec.0000133611.42699.08

Garcia de Blakeley, M., Ford, R., \& Casey, L. (2017). Second language anxiety among Latino American immigrants in Australia. International Journal of Bilingual Education and Bilingualism, 20(7), 759-772. https://doi.org/10.1080/13670050.2015.1083533

Garcia de Blakeley, M. (2020). Cross-lingual competence: Conceptualization, measurement, and relevance to mental health practitioners [unpublished doctoral dissertation]. Griffith University.

Gibbons, J. (1995). What got lost? The place of electronic recording and interpreters in police interviews. In D. Eades (Ed.), Language in evidence: Issues confronting aboriginal and multicultural Australia (pp. 175-186). UNSW Press.

Gold Coast Hospital and Health Service. (2017). Procedure Document PRO0405: Interpreter services bookings and access by patients. Queensland Government.

Grace, K. T., \& Anderson, J. C. (2018). Reproductive coercion: A systematic review. Trauma, Violence, \& Abuse, 19(4), 371-390. https://doi.org/10.1177/1524838016663935

Gray, B., Hilder, J., \& Donaldson, H. (2011). Why do we not use trained interpreters for all patients with limited English proficiency? Is there a place for using family members? Australian Journal of Primary Health, 17, 240-249. https://doi.org/10.1071/ PY10075

Hilder, J., Gray, B., Dowell, A., Macdonald, L., Tester, R., \& Stubbe, M. (2017). 'In depends on the consultation': Revisiting use of family members as interpreters for general practice consultations - when and why? Australian Journal of Primary Health, 23, 257-262. https://doi.org/10.1071/PY16053

Holliday, C. N., McCauley, H. L., Silverman, J. G., Ricci, E., Decker, M. R., Tancredi, D. J., Burke, J. G., Documet, P., Borrero, S., \& Miller, E. (2017). Racial/Ethnic differences in women's experiences of reproductive coercion, intimate partner violence, and unintended pregnancy. Journal of Women's Health, 26(8), 828835. https://doi.org/10.1089/jwh.2016.5996

Huang, Y., \& Phillips, C. (2009). Telephone interpreters in general practice: Bridging the barriers to their use. Australian Family Physician, 38(6), 443-446.

In Touch, (2010). "I lived in fear because I knew nothing": Barriers to the justice system faced by CALD women experiencing family violence. Victoria, Australia.

Javier, R. A. (1995). Vicissitudes of autobiographical memories in a bilingual analysis. Psychoanalytic Psychology, 12(3), 429-438. https://doi.org/10.1037/h0079703

Jones, L., Sheeran, N., Pines, R., \& Saunders, B. (2019). How do health professionals decide whether an interpreter is needed for families in neonatal and pediatric units? Patient Education and Counselling, 102(9), 1629-1635. https://doi.org/10.1016/j.pec.2019.04. 004

Judicial Council on Cultural Diversity. (2016). The Path to Justice: Migrant and Refugee Women's Experience of the Courts. Canberra, Australia.

Karliner, L. S., Jacobs, E. A., Chen, A. H., \& Mutha, S. (2007). Do professional interpreters improve clinical care for patients with limited English proficiency? A systematic review of the literature.
Health Services Research, 42, 727-754. https://doi.org/10.1111/j. 1475-6773.2006.00629.x

Lemon, N. K. (2006). Access to justice: Can domestic violence courts better address the need of non-English speaking victims of domestic violence. Berkeley Journal of Gender, Law \& Justice, $21,38-58$

Miller, E., Decker, M. R., McCauley, H. L., Tancredi, D. J., Levenson, R. R., Waldman, J., . . . Silverman, J. G. (2010). Pregnancy coercion, intimate partner violence and unintended pregnancy. Contraception, 81(4), 316-322. https://doi.org/10.1016/j.contr aception.2009.12.004

Minas, H., Kakuma, R., Too, L. S., Vayani, H., Orapeleng, S., PrasadIldes, R., Turner, G., Procter, N., \& Oehm, D. (2013). Mental health research and evaluation in multicultural Australia: Developing a culture of inclusion. International Journal of Mental Health Systems, 7(1), 23. https://doi.org/10.1186/1752-4458-7-23

Mitra-Kahn, T., Newbigin, C., \& Hardefeldt, S. (2016). Invisible women, invisible violence: Understanding and improving data on the experiences of domestica and family violence and sexual assault for diverse groups of women: State of knowledge paper (ANROWS Landscapes, DD01/2016). ANROWS.

Molina, R. L., \& Kasper, J. (2019). The power of language-concordant care: A call to action for medical schools. BMC Medical Education, 19(1), 378. https://doi.org/10.1186/s12909-019-1807-4

Nancarrow, H., Thomas, K., Ringland, V., \& Modini, T. (2020). Accurately identifying the "person most in need of protection" in domestic and family violence law (Research report, 23/2020). ANROWS.

Pavlenko, A. (2006). Bilingual minds: Emotional experience, expression, and representation. Multilingual Matters.

Phillips, C. (2010). Using interpreters: A guide for GPs. Australian Family Physician, 39(4), 188-195.

Pines, R., Jones, L., \& Sheeran, N. (2020). Using family members as medical interpreters: An explanation of healthcare practitioners' normative practices in Pediatric and Neonatal departments in Australia. Health Communication, 35(7), 902-909. https://doi.org/10. 1080/10410236.2019.1598740

Price, E., Sharman, L. S., Douglas, H. A., Sheeran, N., \& Dingle, G. A. (2019). Experiences of reproductive coercion in Queensland women. Journal of Interpersonal Violence. https://doi.org/10. $1177 / 0886260519846851$

Raj, A., \& Silverman, J. (2002). Violence against immigrant women: The roles of culture, context, and legal immigrant status on intimate partner violence. Violence Against Women, 8(3), 367-398. https://doi.org/10.1177/10778010222183107

Rowlands, S., \& Walker, S. (2019). Reproductive control by others: Means, perpetrators and effects. BMJ Sexual and Reproductive Health, 45, 61-67.

Schwanberg, J. S. (2010). Does language of retrieval affect the remembering of trauma? Journal of Trauma \& Dissociation, 11(1), 44-56. https://doi.org/10.1080/15299730903143550

Silverman, J. G., \& Raj, A. (2014). Intimate partner violence and reproductive coercion: Global barriers to women's reproductive control. PLoS Medicine, 11(9), e1001723. https://doi.org/10. 1371/journal.pmed.1001723

Smith, O., \& Skinner, T. (2017). How rape myths are used and challenged in rape and sexual assault trials. Social \& Legal Studies, 26(4), 441-466. https://doi.org/10.1177/0964663916680130

Stark, E. (2007). Coercive Control: How Men Entrap Women in Personal Life. Oxford University Press.

Stark, E., \& Hester, M. (2019). Coercive control: Update and review. Violence against Women, 25(1), 81-104. https://doi.org/10. $1177 / 1077801218816191$

Tam, Tutty, Zhuang, \& Paz. (2016). Racial minority women and criminal justice responses to domestic violence. Journal of 
Family Violence, 31(4), 527-538. https://doi.org/10.1007/ s10896-015-9794-7

Tarzia, L., \& Hegarty, K. (2021). A conceptual re-evaluation of reproductive coercion: Centring intent, fear and control. BMC Reproductive Health, 18, 87. https://doi.org/10.1186/ s12978-021-01143-6

Tarzia, L., Douglas, H., \& Sheeran, N. (2021). Reproductive coercion and abuse against women from migrant and refugee backgrounds: Exploring the views of service providers in Australia. Culture, Health and Sexuality. https://doi.org/10.1080/13691 058.2020 .1859617

Taylor, N., \& Putt, J. (2007). Adult sexual violence in Indigenous and culturally and linguistically diverse communities in Australia. Trends \& issues in crime and criminal justice no. 345. Canberra: Australian Institute of Criminology. https://www.aic.gov.au/publi cations/tandi/tandi345. Accessed 2 Dec 2020.

Ulbrick, M., \& Jago, M. (2018). "Officer she's psychotic and I need protection": Police misidentification of the "primary aggressor" in family violence incidents in Victoria (Policy paper no. 1). Victoria: Women's Legal Service Victoria and Monash University.
Vaughan, C., Davis, E., Murdolo, A., Chen, J., Murray, L., Block, K., Quiazon, R., Warr, D. (2016). Promoting community-led responses to violence against immigrant and refugee women in metropolitan and regional Australia: The ASPIRE Project: Key findings and future directions. ANROWS Compass: Research to policy and practice (8). Sydney: ANROWS.

Wakefield, S. J., Kebbell, M. R., Moston, S., \& Westera, N. (2015). Perceptions and profiles of interviews with interpreters: A police survey. Australia \& New Zealand Journal of Criminology, 48(1), 53-72. https://doi.org/10.1177/0004865814524583

Wellington, M., Hegarty, K., \& Tarzia, L. (2021). Barriers to responding to reproductive coercion and abuse among women presenting to Australian primary care. BMC Health Services Research, 21, 424. https://doi.org/10.1186/s12913-021-06420-5

Publisher's Note Springer Nature remains neutral with regard to jurisdictional claims in published maps and institutional affiliations. 